\title{
A Fundamental Study of Supercritical Flow at Sudden Expansion Structure by Using Numerical Model
}

\author{
Jia Jun Lim ${ }^{1}$, How Tion Puay ${ }^{*}$ and Nor Azazi Zakaria ${ }^{1}$ \\ ${ }^{1}$ River Engineering and Urban Drainage Research Centre (REDAC), Universiti Sains Malaysia, Seri \\ Ampangan, 14300 Nibong Tebal, Penang, Malaysia.
}

\begin{abstract}
In the design of hydraulic structures, it is common to deal with the supercritical flow. Abrupt expansion structure represents a type of transition often constructed in manmade hydraulic structure (e.g. chutes in dam structures, sewer or drainage system, an irrigation channel, etc.) to cater the geometry difference. It is important to cater to the design of such transition especially it involves flow with high velocity. The flow features of high-velocity flow at sudden expansion channel are studied. A twodimensional depth-averaged model (DA model) incorporate with Constrained Interpolation Profile (CIP) scheme is developed to simulate the supercritical flow at the abrupt expansion channel. The numerical model is able to reproduce the flow features of supercritical flow at the abrupt expansion structure. The simulated results are compared with the analytical solution with a different degree of agreement is observed. The same numerical setup is applied to the model generated by FLOW-3D model. The numerical results from the DA model and FLOW-3D model are compared with previous experimental results. The simulated results from the DA model showed better agreement with the experimental results.
\end{abstract}

\section{Introduction}

A transition structure introduced in open channel represents a change in the cross-section, direction or slope in the channel. It is widely used in the hydraulic structures such as sluice gates, steep chutes, sewer system and sometimes open channel involved in irrigation purpose. Sudden expansion or abrupt expansion can be defined as the sudden increase in the crosssectional flow area of the channel in the direction of flow. Supercritical flow often associates with such transition in the open channel and cause the formation of oblique stationary waves. These waves may overtop the side wall, causing damage to the hydraulic structure and the surrounding. A proper design of the abrupt expansion transition structure is thus crucial [1].

The state of flow whether it is supercritical or subcritical is affected by gravity, it is represented by the ratio of inertial forces to gravity forces, commonly known as Froude number, Fr. Whenever the Froude number of a flow is less than one, the flow is subcritical. If it is equal to one, the flow is in a critical state. If the Froude number is greater than one,

\footnotetext{
${ }^{*}$ Corresponding author: redac puay@usm.my
} 
then the flow is said to be supercritical. In this state, the effect of inertial forces is more pronounced, the flow usually has a high velocity [2].

In 1951, Rouse et al. [1] conducted a series of experiments on open channel expansion, it was considered as the pioneering research in channel expansion. In contrast with the ordinary empirical analysis done on such a specific problem, Rouse et al. [1] incorporated the elementary wave theory to the analysis of supercritical flow in channel expansion. The study related the surface profile of supercritical flow in abrupt expansion channel with a various Froude number and width-depth ratio involved. The results were presented in the generalized diagram form for convenient in preliminary design. Rouse et al. [1] concluded that the result from the experiments of supercritical flow in open channel expansion shows a close agreement with the elementary wave theory if the assumptions involved are approximately satisfied.

Hager and Mazumder [3] studied the phenomenon of high-velocity flow in abrupt expansion channel using a semi-empirical approach. The study showed that both the axial and transverse profiles are independent of the expansion ratio between the approach channel and the tailwater channel.

By employing the method of characteristics, Hosoda and Yoneyama [4] developed an analytical solution for the abrupt channel flow, the angle of shock waves and the angle of flow expansion. The authors further relate the effect of different Froude number with the change in direction of high-velocity flow.

This study demonstrates a two-dimensional depth-averaged model (DA model) to simulate the phenomenon of high-velocity flow at the sudden expansion channel. Constrained Interpolation Profile (CIP) scheme [5] is employed to solve the advection part in the momentum equation, where this scheme is of third order accuracy.

\section{Flow characteristics of high-velocity flow at abrupt expansion channel}

When high-velocity flow occurring at the sudden expansion channel, the flow emerging from the expansion subjected to a change in direction through an angle of flow expansion, $\theta$ (Figure 1). The flow will then impinge at the side wall of the tailwater channel at the impingement points, forming oblique standing waves at an angle of the shock wave, $\beta$ (Figure 1). These waves continue undiminished in the downstream direction couples with large surface disturbances [6].

There is a region at the abrupt expansion where the flow depth and velocity are constant and equivalent to the approach flow depth, $h_{0}$ and approach velocity, $v_{l}$. This triangular region is known as the zone of constant depth and velocity [4]. 


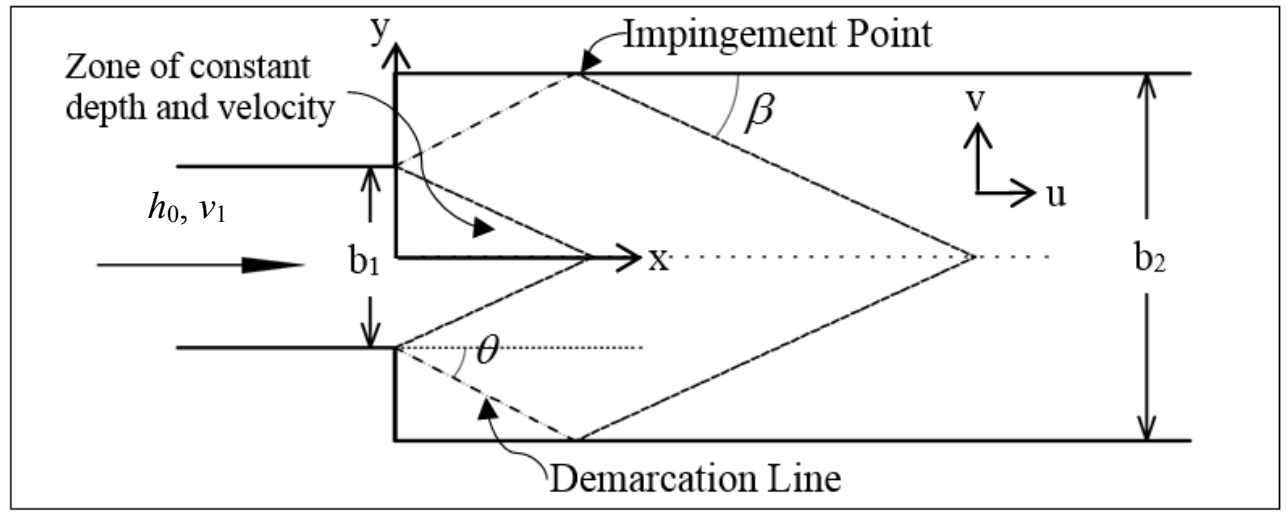

Fig. 1. Flow features of high-velocity flow at sudden expansion channel.

\section{Analytical study}

The following governing equations describe the two-dimensional depth-averaged model (DA model) for the problem of high-velocity flow at sudden expansion structures in the condition of steady state:

Continuity equation:

$\underline{\text { Momentum equation: }}$

$$
\begin{gathered}
\frac{\partial h u}{\partial x}+\frac{\partial h v}{\partial x}=0 \\
\frac{\partial h u^{2}}{\partial x}+\frac{\partial h v}{\partial y}=-g h \frac{\partial h}{\partial x}+g h \sin \alpha-\frac{\tau_{b x}}{\rho} \\
\frac{\partial h v}{\partial x}+\frac{\partial h v^{2}}{\partial y}=-g h \frac{\partial h}{\partial y}-\frac{\tau_{b y}}{\rho}
\end{gathered}
$$

Where $u$ and $v$ are the components of depth-averaged velocity vectors in $x$ and $y$-direction, $\tau_{b y}$ and $\tau_{b y}$ are the components of bottom shear stress vectors in $x$ and $y$-direction, $\alpha$ is the angle between the channel bottom and the horizontal datum, $\rho$ is the density of water, $h$ is the flow depth and $g$ is the acceleration due to gravity.

By using the governing equations, Ippen [6] derived a relationship to describe the angle of expansion, $\theta$

$$
\theta=\sqrt{3} \tan ^{-1} \frac{\sqrt{3}}{\sqrt{F r^{2}-1}}-\tan ^{-1} \frac{1}{\sqrt{F r^{2}-1}}-\theta_{1}
$$

In which $\theta_{1}$ is a constant which defined with the condition when $\theta=0$ and the flow depth is represented by the initial depth.

In the case where the depth difference across the shock wave is small, the angle of the shock wave $\beta$ is given in simplified form [6]:

$$
\beta=\sin ^{-1} \frac{1}{F r}
$$

\section{Numerical analysis}

\subsection{Governing equation}

The two-dimensional depth-averaged model (DA model) is developed based on the following governing equation in their non-conservative form. 
Continuity Equation:

$$
\frac{\partial h}{\partial t}+u \frac{\partial h}{\partial x}+v \frac{\partial h}{\partial y}=-h\left(\frac{\partial u}{\partial x}+\frac{\partial v}{\partial y}\right)
$$

Momentum Equation:

$$
\begin{gathered}
\frac{\partial u}{\partial t}+u \frac{\partial u}{\partial x}+v \frac{\partial u}{\partial y}=-g\left(\frac{\partial h}{\partial x}+\frac{\partial z_{b}}{\partial x}\right)-\frac{g n^{2} u \sqrt{u^{2}+v^{2}}}{h^{1 / 3}} \\
\frac{\partial v}{\partial t}+u \frac{\partial v}{\partial x}+v \frac{\partial v}{\partial y}=-g\left(\frac{\partial h}{\partial y}+\frac{\partial z_{b}}{\partial y}\right)-\frac{g n^{2} v \sqrt{u^{2}+v^{2}}}{h^{1 / 3}}
\end{gathered}
$$

Where $u$ and $v$ are the components of depth-averaged velocity vectors in $x$ and $y$-direction, $h$ is the flow depth, $n$ is the Manning's Coefficient, $z_{b}$ is the vertical elevation of the channel bed from a horizontal reference datum and $g$ is the acceleration due to gravity.

\subsection{Numerical algorithm}

In the case of an inviscid fluid, bed shear stresses were neglected, and Eq. 7 and Eq. 8 become,

$$
\begin{aligned}
& \frac{\partial u}{\partial t}+u \frac{\partial u}{\partial x}+v \frac{\partial u}{\partial y}=-g\left(\frac{\partial h}{\partial x}+\frac{\partial z_{b}}{\partial x}\right)=-g \frac{\partial h}{\partial x} \\
& \frac{\partial v}{\partial t}+u \frac{\partial v}{\partial x}+v \frac{\partial v}{\partial y}=-g\left(\frac{\partial h}{\partial y}+\frac{\partial z_{b}}{\partial y}\right)=-g \frac{\partial h}{\partial y}
\end{aligned}
$$

Where in the case of flat bed, $\frac{\partial z_{b}}{\partial x}=\frac{\partial z_{b}}{\partial y}=0$.

In solving the governing equation, the time-splitting method [5] is used. Eq. 6, 7 and 8 are solved in two stages, namely the advection stage and non-advection stage,

$$
\begin{aligned}
& \frac{\text { Advection stage: }}{\partial h} \frac{\partial h}{\partial t}+u \frac{\partial h}{\partial x}+v \frac{\partial}{\partial y}=0 \\
& \frac{\partial u}{\partial t}+u \frac{\partial u}{\partial x}+v \frac{\partial u}{\partial y}=0 \\
& \frac{\partial v}{\partial t}+u \frac{\partial v}{\partial x}+v \frac{\partial v}{\partial y}=0
\end{aligned}
$$

$$
\begin{aligned}
& \frac{\text { Non-advection stage: }}{\partial h} \frac{\partial u}{\partial t}=-h\left(\frac{\partial u}{\partial x}+\frac{\partial v}{\partial x}\right) \\
& \frac{\partial u}{\partial t}=-g \frac{\partial h}{\partial x} \\
& \frac{\partial v}{\partial t}=-g \frac{\partial h}{\partial y}
\end{aligned}
$$

The numerical algorithm is summarized as follows [7]:

First, in the advection stage, the advection terms of the continuity and momentum equations Eq. 11, 12 and 13 are solved using the Constrained Interpolation Profile (CIP) scheme. Temporary values of $h^{*}, u^{*}$ and $v^{*}$ are obtained after the advection terms are solved.

$$
\begin{array}{lll}
\frac{\partial h}{\partial t}+u \frac{\partial h}{\partial x}+v \frac{\partial h}{\partial y}=0 & \longrightarrow & h^{n} \stackrel{C I P}{\rightarrow} h^{*} \\
\frac{\partial u}{\partial t}+u \frac{\partial u}{\partial x}+v \frac{\partial u}{\partial y}=0 & \longrightarrow & u^{n} \stackrel{C I P}{\rightarrow} u^{*} \\
\frac{\partial v}{\partial t}+u \frac{\partial v}{\partial x}+v \frac{\partial v}{\partial y}=0 & \longrightarrow & v^{n} \stackrel{C I P}{\rightarrow} v^{*}
\end{array}
$$

In the non-advection stage, the non-advection term, Eq. 14,15 and 16 are solved using the finite difference method and discretization is based on the staggered mesh system.

$$
\begin{array}{ll}
\frac{\partial h}{\partial t}=-h\left(\frac{\partial u}{\partial x}+\frac{\partial v}{\partial x}\right) & \rightarrow \frac{h^{n+1}-h^{*}}{\Delta t}=-h^{*}\left(\frac{\partial u^{n+1}}{\partial x}+\frac{\partial v^{n+1}}{\partial y}\right) \\
\frac{\partial u}{\partial t}=-g \frac{\partial h}{\partial x} & \longrightarrow \frac{u^{n+1}-u^{*}}{\Delta t}=-g \frac{\partial}{\partial x}\left(h^{n+1}\right) \\
\frac{\partial v}{\partial t}=-g \frac{\partial h}{\partial y} & \longrightarrow \frac{v^{n+1}-v^{*}}{\Delta t}=-g \frac{\partial}{\partial y}\left(h^{n+1}\right)
\end{array}
$$

From Eq. 21 and 22, the value $u^{n+1}$ and $v^{n+1}$ are calculated explicitly. The following equations were formulated where the initial guess of $u^{n+1}$ and $v^{n+1}$ are labeled as $\tilde{u}$ and $\tilde{v}$. 


$$
\begin{aligned}
& \tilde{u}=u^{*}-g \Delta t \frac{\partial}{\partial x}\left(h^{*}\right) \\
& \tilde{v}=v^{*}-g \Delta t \frac{\partial}{\partial y}\left(h^{*}\right)
\end{aligned}
$$

Subtracting Eq. 23 from Eq. 21, and Eq. 24 from Eq. 22, forms the following equation,

$$
\begin{array}{r}
\frac{u^{n+1}-\tilde{u}}{\Delta t}=-g \frac{\partial(\delta h)}{\partial x} \\
\frac{v^{n+1}-\tilde{v}}{\Delta t}=-g \frac{\partial(\delta h)}{\partial y} \\
\text { Where } \delta h=h^{n+1}-h^{*}
\end{array}
$$

Eq. 25 and 26 are substituted into Eq. 20 to obtain Pothe isson equation as follows,

$$
\frac{\partial^{2}(\delta h)}{\partial x^{2}}+\frac{\partial^{2}(\delta h)}{\partial y^{2}}=\frac{1}{g h^{*}(\Delta t)^{2}} \delta h+\frac{1}{g \Delta t}\left(\frac{\partial \tilde{u}}{\partial x}+\frac{\partial \widetilde{v}}{\partial y}\right)
$$

The Poisson equation is solved using the Successive Over Relaxation (SOR) method to obtain $\delta h$ and the values of $h, u$ and $v$ are updated using Eq. 25, 26 and 27.

$$
\begin{gathered}
h^{n+1}=h^{*}+\delta h \\
u^{n+1}=\tilde{u}-g \Delta t \frac{\partial}{\partial x}(\delta h) \\
v^{n+1}=\tilde{v}-g \Delta t \frac{\partial}{\partial y}(\delta h)
\end{gathered}
$$

\section{Numerical analysis}

\subsection{Verification of the numerical model}

\subsubsection{Dam break problem (One-dimensional)}

A one-dimensional dam break flow problem over a dry bed condition is being simulated to test the accuracy and numerical stability of the depth-averaged model (DA model). In this simulation, an idealized case of a dam break scenario is considered. The DA model simulates the occurrence of the dam break when the vertical wall diving two different water depths is suddenly removed. The DA model is used to simulate the dam break problem of a domain range from $0 \mathrm{~m} \leq x \leq 15.0 \mathrm{~m}$ with the following initial conditions:

$$
h(x, t=0)=\left\{\begin{array}{l}
1.5 \mathrm{~m}, 0 \mathrm{~m} \leq x \leq 5.0 \mathrm{~m} \\
0.0 \mathrm{~m}, 5 \mathrm{~m}<x \leq 15.0 \mathrm{~m}
\end{array} \quad ; \quad u(x, t=0)=0.0 \mathrm{~m} / \mathrm{s}\right.
$$

Figure 2 shows the comparison of the simulated solution with Ritter's solution [8]. The results from DA models are indicated with filled circle while Ritter's solution is representing with a solid line in Figure 2. The dashed line shows the initial condition mentioned. The numerical result has good agreement with the Ritter's solution.

\subsubsection{Partial dam break problem (Two-dimensional)}

The DA model is used to simulate a two-dimensional partial dam break problem with the following initial conditions:

$$
h(x, t=0)=\left\{\begin{array}{c}
10.0 \mathrm{~m}, \quad 0.0 \mathrm{~m} \leq x \leq 100.0 \mathrm{~m} \\
5.0 \mathrm{~m}, \quad 100.0 \mathrm{~m}<x \leq 200.0 \mathrm{~m}
\end{array} \quad ; \quad \begin{array}{l}
u(x, y, t=0)=0.0 \mathrm{~m} / \mathrm{s} \\
v(x, y, t=0)=0.0 \mathrm{~m} / \mathrm{s}
\end{array}\right.
$$




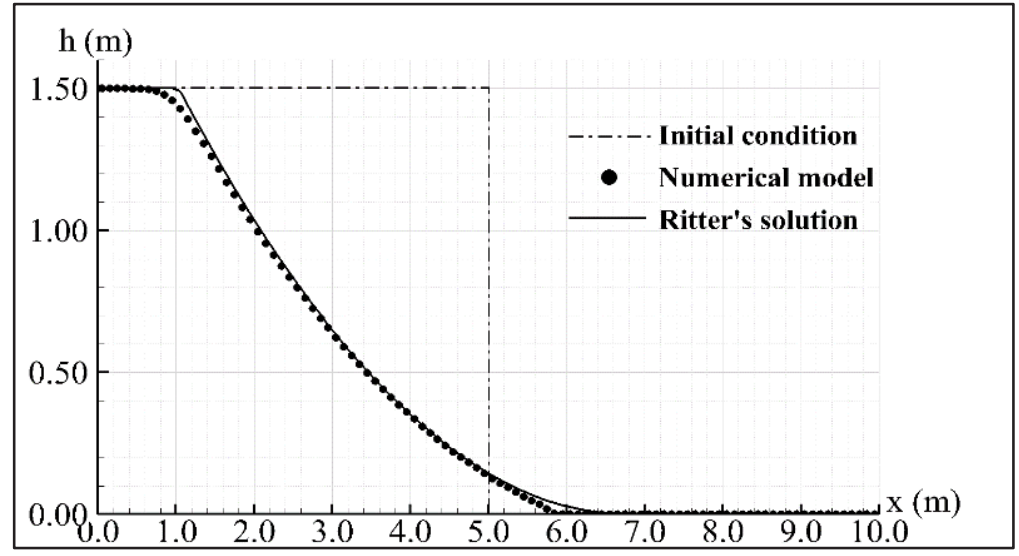

Fig. 2. Comparison of numerical solution and Ritter's solution for dam break flow over dry bed condition.

(a)

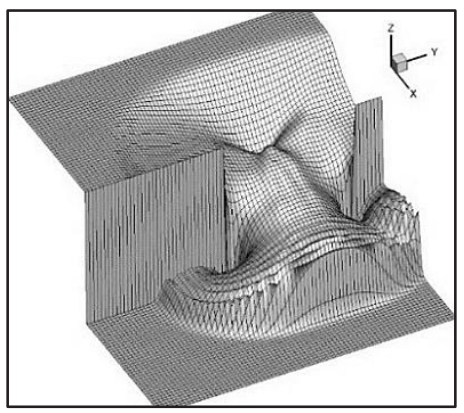

(b)

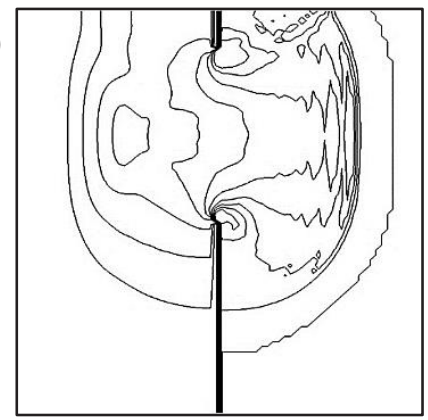

Fig. 3. Numerical solution of water depth as viewed from (a) three-dimensional perspective and (b) contour perspective.

(a)

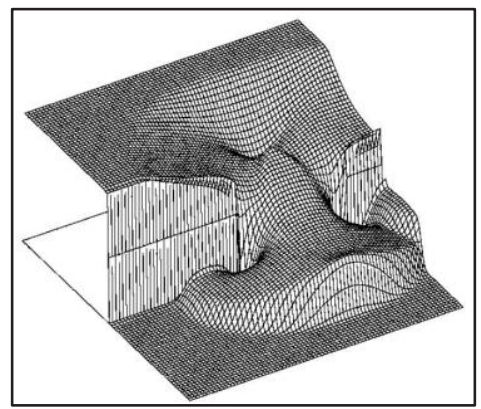

(b)

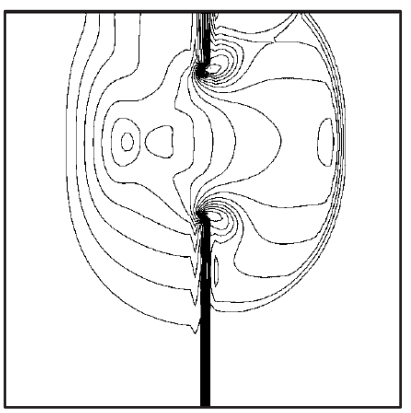

Fig. 4. Numerical simulation of water depth from Akoh et al. [9] studies at as viewed from (a) threedimensional perspective and (b) contour perspective.

The numerical solution from the DA model shows comparable simulation accuracy when compared with Akoh et al. [9] studies (Figure 4).

\section{Numerical results}

The two-dimensional depth-averaged model (DA model) is used to simulate the occurrence of supercritical flow at abrupt expansion transition in a rectangular channel. Section 6.1 describes the comparison between the numerical simulation and analytical solution. In which 
inviscid flow condition was introduced to comply with the assumption made when deriving the analytical solution. Section 6.2 describes the numerical results from DA model and FLOW-3D software, and the comparison with the experimental results from Hager and Mazumder [3] research.

\subsection{Comparison between numerical solution and analytical solution}

Table 1. Simulation set up for comparing Numerical Solution and Analytical Solution.

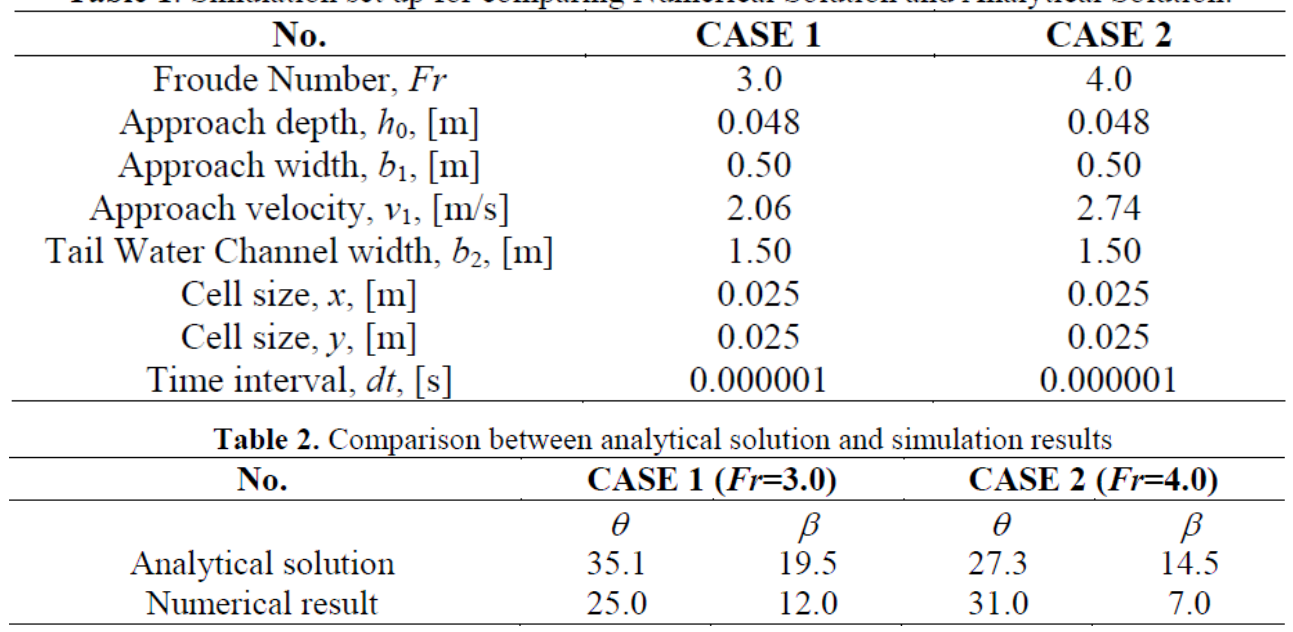

The DA model is used to simulate two scenarios of different conditions set up as shown in Table 1. Two different flow conditions with $F r=3.0$ and $F r=4.0$ are simulated in this part of the study. The flow is allowed to discharge from the approach channel (i.e. the location of $b_{1}$ ) into a tailwater channel of width $1.50 \mathrm{~m}$ passing through a supercritical transition.

The DA model can simulate the supercritical flow features at abrupt expansion channel as mentioned in Section 2, the simulation results are shown in Figure 5 and 6. The angle of flow expansion, $\theta$ and the angle of the shock wave, $\beta$ obtained from the DA model are then compared with the analytical solution based on Eq. 4 and 5. The comparison is summarised in Table 2.

In figures 5 and 6 , the solid line represents the numerical solution simulated from the DA model, while the analytical solution is denoted with a dashed line.

For CASE $1(F r=3.0)$, the angle of expansion, $\theta$ determined from the analytical solution is $35.1^{\circ}$, whereas the results generated from the DA model is $25.0^{\circ}$ (Figure 5). For CASE 2 $(F r=4.0)$, the agreement of angle $\theta$ between the DA model $\left(\theta=31.0^{\circ}\right)$ and the analytical solution $\left(\theta=27.3^{\circ}\right)$ is fairly good (Figure 6). These show that the demarcation line determined from the numerical simulation (DA model) has a satisfactory agreement with the analytical solution.

For the angle of shock waves, $\beta$, the result obtained from the numerical simulation is $12.0^{\circ}$ while the result determined from the analytical solution is $19.5^{\circ}$. These show a satisfactory agreement between these two approaches, as shown in Figure 5. While in Figure 6 , the angle of shock waves $\beta$ from the numerical model is $7.0^{\circ}$ and the analytical solution is $14.5^{\circ}$. The agreement between the numerical model and the analytical solution is unsatisfactory. These suggest that the DA model could reproduce the angle of flow expansion, $\theta$ and shock wave angle, $\beta$ with varying accuracies. This is due to the difficulty to determine the exact demarcation lines (for the determination of $\theta$ ) and wave starting location (for the determination of $\beta$ ). 


\subsection{Comparison of numerical solution and experimental results [3]}

Table 2 shows the numerical set up of the DA model and FLOW-3D model where the Real fluid conditions were applied in the simulations to compare with the experimental results [3].

Table 3. Simulation set up for comparing numerical solution with experimental results.

\begin{tabular}{ccc}
\hline No. & CASE 3 & CASE 4 \\
\hline Froude Number, $F r$ & 4.0 & 6.0 \\
Approach depth, $h_{0},[\mathrm{~m}]$ & 0.048 & 0.048 \\
Approach width, $b_{1},[\mathrm{~m}]$ & 0.50 & 0.50 \\
Approach velocity, $v_{1},[\mathrm{~m} / \mathrm{s}]$ & 2.75 & 4.12 \\
Tail Water Channel width, $b_{2},[\mathrm{~m}]$ & 1.50 & 1.50 \\
Manning's Roughness Coefficient, $n$ & 0.010 & 0.010 \\
Cell size, $x,[\mathrm{~m}]$ & 0.025 & 0.025 \\
Cell size, $y,[\mathrm{~m}]$ & 0.025 & 0.025 \\
Time interval, $d t,[\mathrm{~s}]$ & 0.000001 & 0.000001 \\
\hline
\end{tabular}
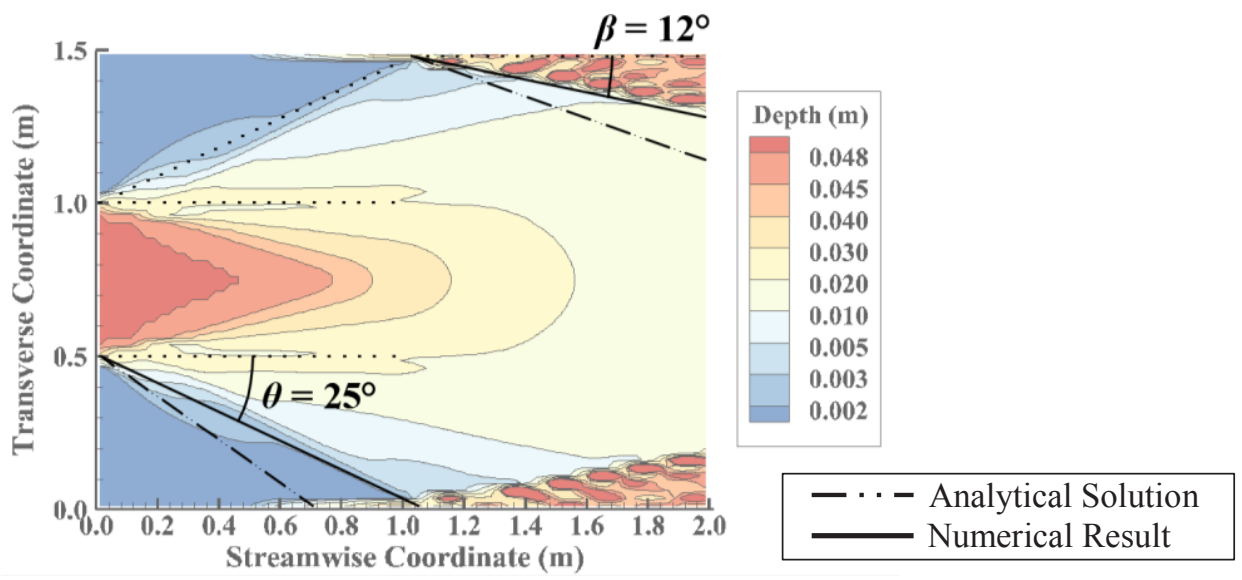

Fig. 5. Simulation result for flow with $F r=3.0$ (plan view) and the comparison between analytical and numerical results.

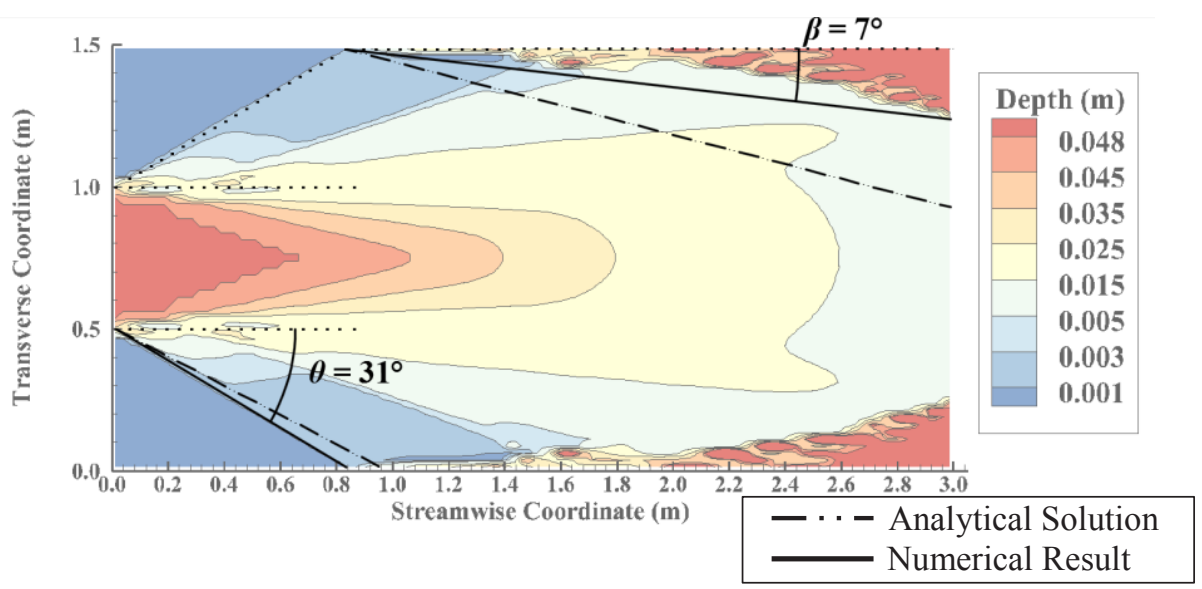


Fig. 6. Simulation result for flow with $F r=4.0$ (plan view) and the comparison between analytical and numerical results.
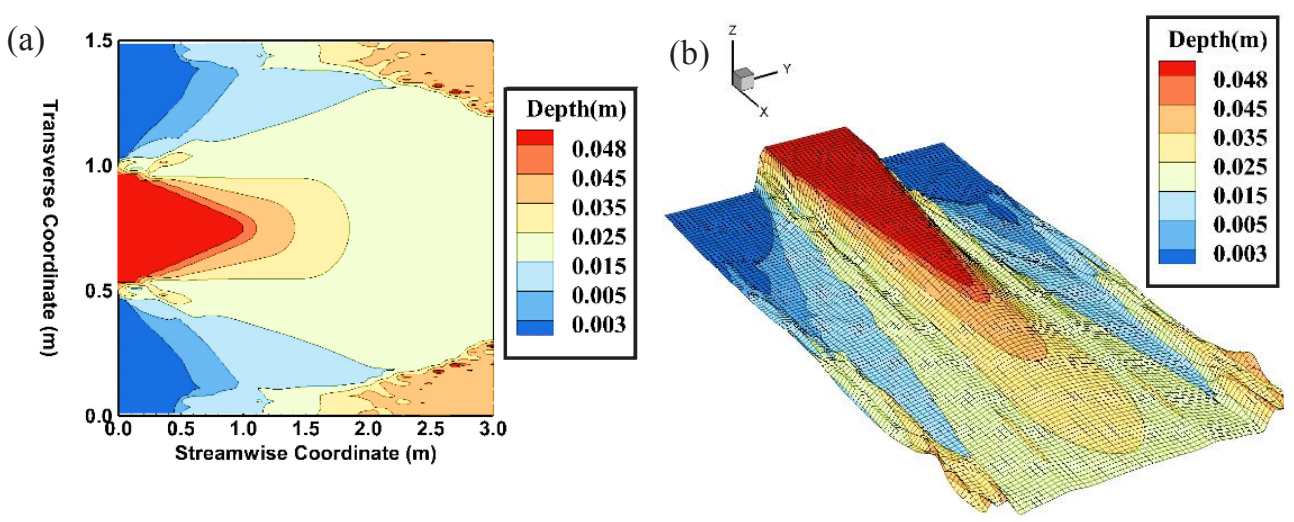

Fig. 7. Simulated result for CASE 3: (a) contour perspective and (b) three-dimensional perspective

(a)

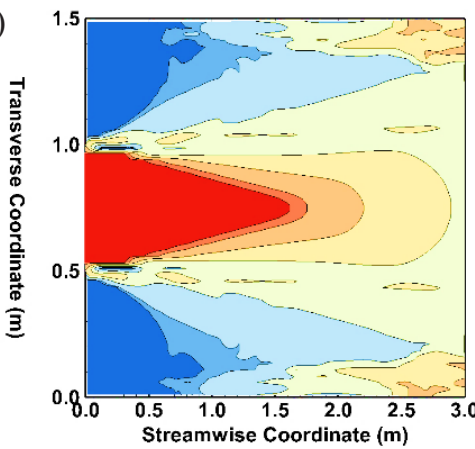

(b) $z$

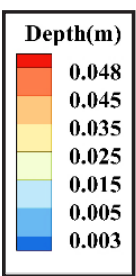

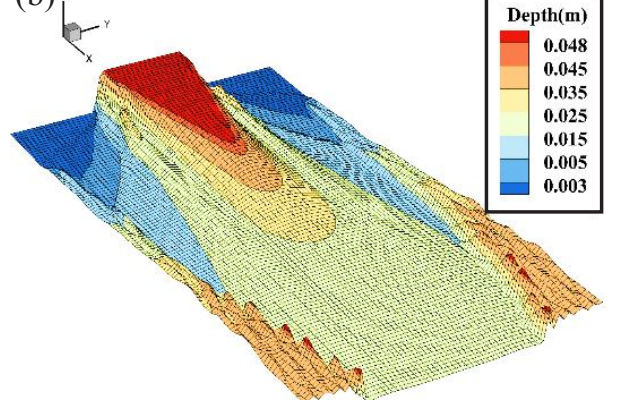

Fig. 8. Simulated result for CASE 4: (a) contour perspective and (b) three-dimensional perspective

Figure 7 and 8 show the simulated results from the DA model with the numerical set up shown in Table 2. The experiments were carried out in a rectangular sudden expansion channel same as the schematic drawing as shown in Figure 1.

Figure 9 and 10 show the sidewall surface profile simulated from the DA model and FLOW-3D model, as well as the experimental data. 


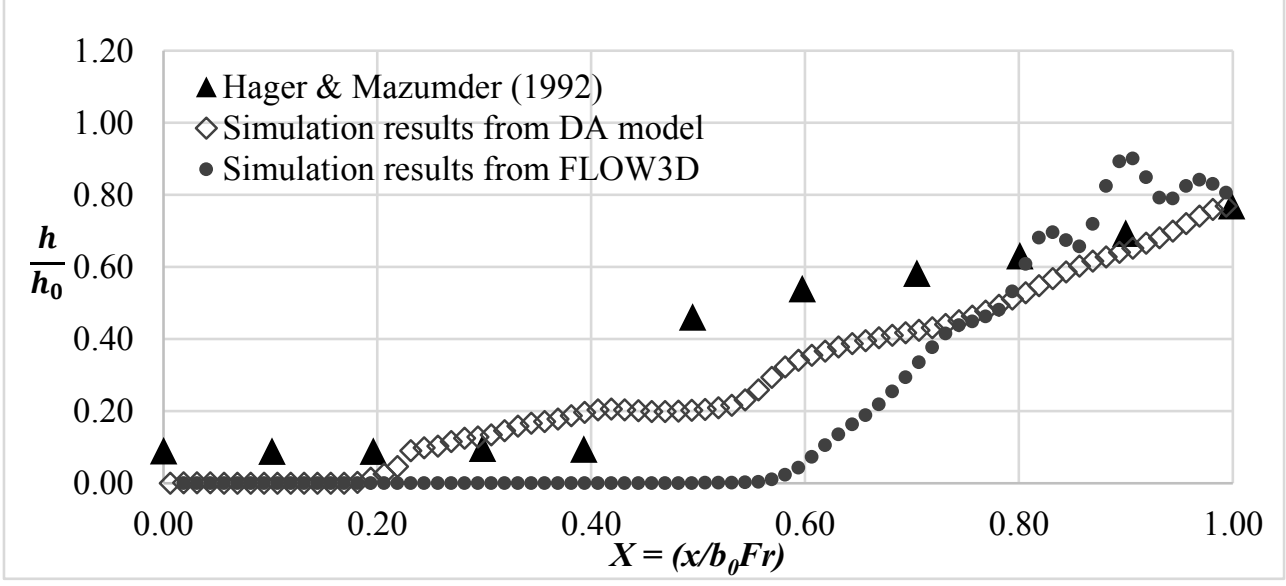

Fig. 9. Sidewall surface profile, $Y_{w}$ for Case S3, where $F r=4, h_{0}=0.048 \mathrm{~m}$

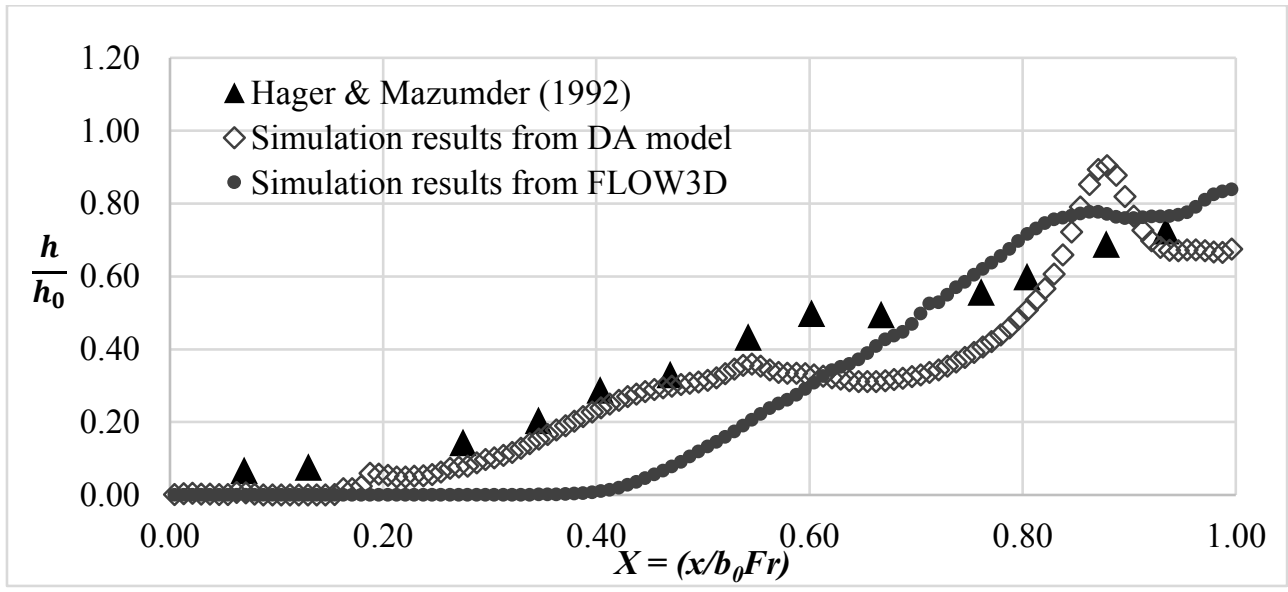

Fig. 10. Sidewall surface profile, $Y_{w}$ for Case $\mathrm{S} 4$, where $F r=6, h_{0}=0.048 \mathrm{~m}$.

The sidewall surface profile, $Y_{w}=h / h_{0}$ is plotted as a function of dimensionless streamwise coordinate, $X=\left(x / b_{0} F r\right)$ where $h$ is the local depth and $x$ is the distance measured from the location of channel expansion

From Figures 9 and 10, the surface profile at sidewall simulated by the DA model shows a close agreement with the experimental data [3]. The high-velocity flow from the expansion travel cross the channel and reflected at the sidewall, this phenomenon results in a hill-shaped zone of flow depth increment at the sidewall. The DA model can simulate this phenomenon and shows good agreement with the experimental results; in the case of $F r=6$ (Figure 10), the results show better agreement.

The surface profile generated by Flow-3D model shows less agreement with the experimental results (Figure 9 and 10). This might due to the first order numerical scheme is incorporated in the FLOW-3D model generated.

\section{Conclusion}

The phenomenon of high velocity at abrupt expansion channel can be reproduced numerically. The flow characteristics of the high-velocity flow at sudden expansion (i.e. the 
zone of constant depth and velocity, core flow and shock wave) can be reproduced well in the depth-averaged model (DA model) employing Constrained Interpolation Scheme (CIP). Comparison of the values of angle of flow expansion, $\theta$, and angle of the shock wave, $\beta$ between the numerical and the analytical solution, show varying degrees of the agreement due to the difficulty to determine demarcation line and wave location in the numerical model. Similarities were observed when comparing the simulated results with experimental data.

\section{References}

1. H. Rouse, B. Bhoota, Trans. ASCE, 116, 347-363 (1951)

2. V. T. Chow, Open channel hydraulics (McGraw-Hill Book Company, New York, 1959)

3. W. H. Hager, S. K. Mazumder, Proc. Instn Civ. Engrs Wat., Marit. \& Energy, 96, 153166 (1992)

4. T. Hosoda, N. Yoneyama, Hydraulic Engineering, 1270-1274 (1994)

5. T. Yabe, Shock waves, 1, 3, 187-195 (1991)

6. A. T. Ippen, Trans. ASCE, 116, 265-400, (1951)

7. K. Kawasaki, T. Ono, N. Piamsa-Nga, A. H. Atsuta, K. Nakatsuji, Proc. of Hydraulic Engineering, 48, 565-570 (2004)

8. H. Chanson, The hydraulics of open channel flow: an introduction (Elsevier Butterworth Heinemann, Oxford, 2004)

9. R. Akoh, S. Ii, F. Xiao, Int J Numer Meth Fl, 56, 12, 2245-2270 (2008) 